\title{
Resultados funcionais do tratamento artroscópico no impacto femoroacetabular em pacientes com mais de 60 anos em comparação com pacientes com 40 anos ou menos*
}

\section{Functional Outcomes of Arthroscopic Treatment in Femoroacetabular Impingement in Patients over 60 Years Old Compared with Patients Aged 40 Years or Younger}

\author{
Bernardo Aguilera-Bohórquez ${ }^{10}$ \\ Erika Cantor ${ }^{3}$ (อ \\ Miguel Brugiatti2 ${ }^{2}$ \\ 1 Unidade de Preservação do Quadril, Instituto de Doenças \\ Osteoarticulares, Centro Médico Imbanaco, Cali, Colômbia \\ 2 Centro Médico Imbanaco, Pontificia Universidad Javeriana de Cali, \\ Cali, Colômbia \\ ${ }^{3}$ Centro Médico Imbanaco, Research Institute, Cali, Colômbia
}

\author{
Ruddy Coaquira ${ }^{2}$
}

Orlando Cardozo 20

\begin{abstract}
Resumo
Palavras-chave

- quadril

- artroscopia

- impacto femoroacetabular

Objetivo Avaliar os resultados funcionais de pacientes diagnosticados com impacto femoroacetabular (IFA) e com mais de 60 anos de idade em comparação aos resultados de pacientes com até 40 anos de idade.

Métodos Esta é uma revisão retrospectiva de pacientes com IFA submetidos à artroscopia do quadril entre 2010 e 2015. Os pacientes eram adultos com mais de 60 anos de idade e Tönnis $\leq 1$, alocados na proporção de 1:1 com adultos de até 40 anos de idade, de acordo com o tipo de deformidade (came, pincer, ou misto), sexo e data de realização da cirurgia.

Resultados Trinta e quatro pacientes foram incluídos em cada grupo. A idade média foi de $30,6 \pm 6,9$ anos e 65,6 $\pm 4,6$ anos nos grupos controle e de casos, respectivamente. Não houve diferenças significativas entre os grupos no acompanhamento de 1 ano $(p>0.05)$. No primeiro ano após a cirurgia, a pontuação Western Ontario and McMaster Universities Osteoarthritis Index (WOMAC) passou de 46,3 para 22,0 no grupo de pacientes mais velhos (casos) e de 38,1 para 7,2 no grupo controle em comparação ao estágio pré-operatório.

Conclusão O grupo de pacientes com até 40 anos de idade apresentou uma mudança considerável na pontuação WOMAC, mas sem significado estatístico em comparação
\end{abstract}

\begin{abstract}
Endereço para correspondência Bernardo Aguilera-Bohórquez, MD, Unidad de Preservación de Caderas del Instituto de Enfermedades Osteoarticulares, Centro Médico Imbanaco, Carrera 38A, Nㅜㄷ-100 Tower A Office 105, Cali, Colombia (e-mail: baguilera@imbanaco.com.co).
\end{abstract}

Trabalho realizado na Unidade de Preservação do Quadril, Instituto de Doenças Osteoarticulares, Centro Médico Imbanaco, Cali, Colômbia. recebido

13 de Dezembro de 2018 aceito

20 de Dezembro de 2019
DOI https://doi.org/ 10.1055/s-0040-1708515. ISSN 0102-3616.
Copyright $\odot 2020$ by Sociedade Brasileira License terms de Ortopedia e Traumatologia. Published by Thieme Revinter Publicações Ltda, Rio de Janeiro, Brazil 
Abstract
Keywords
- hip
- arthroscopy
- femoroacetabular impingement

ao grupo de pacientes acima de 60 anos. Essa observação sugere que a artroscopia do quadril é benéfica quando a seleção de pacientes com IFA é apropriada, independentemente da idade dos indivíduos.

Objective To evaluate the functional outcomes of patients diagnosed with femoroacetabular impingement (FAl) older than 60 years, compared with those of patients of age 40 years or younger.

Methods This was a retrospective review of patients with FAl who underwent hip arthroscopy between 2010 and 2015 . The patients were adults aged over 60 years with Tönnis $\leq 1$ matched in a 1:1 ratio with adults aged 40 years or younger, according to the type of deformity (cam, pincer, or mixed), sex, and the date when the surgery was performed.

Results Thirty-four patients were included in each group. The mean age was $30.6 \pm 6.9$ years and $65.6 \pm 4.6$ years in the control and case groups, respectively. There were no significant differences between the groups at 1-year follow-up $(p>0.05)$. In the group with older patients (case group), we observed a change in the total Western Ontario and McMaster Universities Osteoarthritis Index (WOMAC) score from 46.3 to 22.0 in the $1^{\text {st }}$ postoperative year, while the control cases improved in the WOMAC score from 38.1 to 7.2 in relation to the preoperative stage.

Conclusion In the group of patients $\leq 40$ years old, a considerable change was observed in the WOMAC score without a statistical significance compared with the $>$ 60 years group. This observation suggests that hip arthroscopy is beneficial when there is an appropriate selection of patients with FAl, regardless the age of the patient.

\section{Introdução}

O impacto femoroacetabular(IFA) é reconhecido como um fator predisponente ao desenvolvimento de degeneração precoce do quadril e progressão para osteoartrite (OA) avançada. ${ }^{1-3}$ Avanços na artroscopia do quadril permitiram o diagnóstico e o tratamento de várias patologias dessa articulação, expandindo as indicações para a inclusão de pacientes idosos com dor associada a sinais radiológicos de IFA. ${ }^{4}$

Estudos anteriores, como o de Ben Tov et al., ${ }^{5}$ revelaram a melhora na dor e na funcionalidade após o tratamento artroscópico do IFA em pacientes com mais de 50 anos e OA em grau 0 ou 1 de Tönnis. Philippon et al., ${ }^{6}$ em 3 anos de acompanhamento, relataram a sobrevida articular de $90 \%$ em pacientes com mais de 50 anos, concluindo que indivíduos com espaço articular superior a $2 \mathrm{~mm}$ podem apresentar melhora da dor e da funcionalidade após a artroscopia do quadril.

Embora existam resultados isolados da artroscopia do quadril na população jovem e adulta, poucos estudos comparativos avaliam a funcionalidade nesses dois grupos. ${ }^{7,8}$ Em um estudo retrospectivo, Domb et al. ${ }^{7}$ compararam os resultados clínicos e funcionais de pacientes com 50 anos de idade ou mais aos resultados de pacientes com 30 anos ou menos e observaram pontuações similares em escalas de satisfação.

O objetivo deste estudo foi a avaliação dos resultados funcionais em pacientes diagnosticados com IFA e com mais de 60 anos de idade em comparação a pacientes com até 40 anos de idade. Nossa hipótese foi que pacientes adequadamente selecionados dos dois grupos apresentariam melhora dos sintomas, independentemente de sua faixa etária.

\section{Métodos}

Realizamos um estudo retrospectivo de pacientes diagnosticados com IFA submetidos à cirurgia artroscópica do quadril entre 2010 e 2015, realizada por um único cirurgião (B. A. B.). Adultos com mais de 60 anos de idade, diagnosticados com IFA e sem OA avançada que foram submetidos à artroscopia do quadril durante o período do estudo foram definidos como o grupo de casos. Pacientes com grau 1, 2, ou 3 na classificação Tönnis de OA, doença inflamatória ou metabólica do quadril, displasia residual e histórico de cirurgia ou fratura anterior do quadril foram excluídos.

O grupo controle foi formado por pacientes de até 40 anos de idade, pareados de acordo com o tipo de defeito (pincer, came, ou misto), sexo e data da cirurgia. Quando mais de um controle atendeu aos critérios de inclusão para um caso específico, o escolhido foi o mais jovem. A seleção retrospectiva foi realizada na proporção de $1: 1$.

O diagnóstico do IFA foi baseado em dados clínicos, no teste de flexão, adução, e rotação interna (FADIR, na sigla em inglês) e em alterações morfológicas (came, pincer, ou misto). O registro institucional de artroscopia do quadril foi revisto para identificação de casos e controles (-Fig. 1). Este estudo foi realizado em conformidade com a declaração de Helsinque 


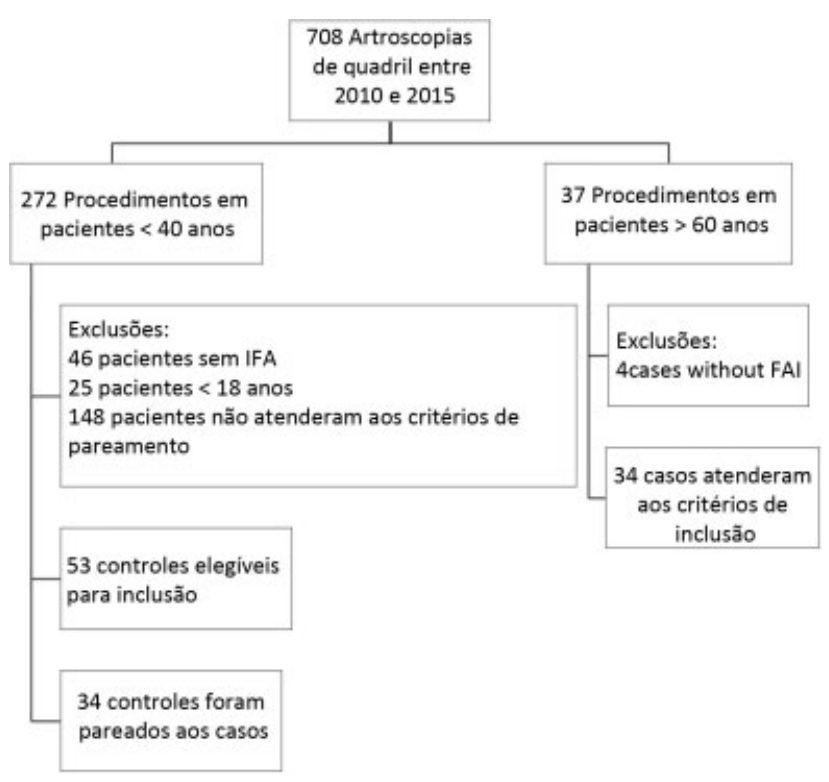

Fig. 1 Fluxograma do processo de seleção do estudo.

e aprovado pelo Comitê de Revisão Institucional (número IRB00008539).

\section{Coleta de Dados}

Dados demográficos, sintomas pré-operatórios, amplitude de movimento (flexão, rotação interna e externa) e pontuação de acordo com o Western Ontario and McMaster Universities Osteoarthritis Index (WOMAC) foram coletados do prontuário clínico. Todos os pacientes foram avaliados de acordo com WOMAC, em três dimensões: dor, rigidez e funcionalidade antes da cirurgia e em 1 ano de acompanhamento. A identificação dos pacientes com necessidade de artroplastia total de quadril ou procedimento de revisão foi realizada por contato telefônico e análise do prontuário médico.

\section{Procedimento Cirúrgico}

Antes do procedimento, todos os pacientes foram avaliados com manobras de provocação (FADIR: flexão, adução e rotação interna) e medidas radiográficas: ângulo $\alpha$ e centro-borda lateral (LCE, na sigla em inglês). O procedimento cirúrgico foi realizado com o paciente em esquema anestésico balanceado (raquianestesia e anestesia geral) em uma mesa ortopédica de tração (MAQUET, Gmbh, Rastatt, Alemanha) e posicionamento em decúbito dorsal.

O compartimento central e periférico do quadril foi acessado pelos portais artroscópicos padrões. O procedimento começou pelo portal anterolateral para a introdução de lentes de $30^{\circ}$ ou $70^{\circ}$ usando a técnica de fora para dentro ou de dentro para fora. A técnica de tenossuspensão foi utilizada para melhorar a visualização da região supra-acetabular ${ }^{9}$ e identificar as áreas de lesões do tipo pincer para acetabuloplastia. Dependendo do grau de lesão labral, até três suturas foram realizadas pelo portal acessório anterolateral inferior.

A seguir, o quadril foi posicionado em flexão de $30^{\circ}$ para alcançar a área de came na junção cabeça-colo e uma capsu- lotomia do ligamento iliofemoral foi realizada para dar continuidade à osteocondroplastia. Por fim, uma manobra dinâmica avaliou as áreas de conflito e a estabilidade do quadril.

\section{Protocolo de Reabilitação}

A partir do segundo dia pós-operatório, os movimentos de flexão do quadril (amplitude de movimento [ADM] ativa e passiva de $0^{\circ}$ a $70^{\circ}$ ) e circunferência foram iniciados em casa, seguidos por caminhada com auxílio de muletas axilares de acordo com a tolerância do paciente.

O protocolo de reabilitação física começou após a segunda semana pós-operatória e foi dividido em 3 fases, com 20 sessões cada ( 5 sessões por semana). Na primeira fase, os pacientes começaram a andar com dispositivos auxiliares para impedir a extensão do quadril operado e proteger o tecido reparado. Na segunda fase, os pacientes caminharam sem muletas e realizaram exercícios progressivos de rotação externa no quadril submetido ao reparo cirúrgico. Por fim, na terceira fase, alguns exercícios de fortalecimento muscular foram iniciados para melhorar a estabilidade e a propriocepção do quadril.

\section{Análises Estatísticas}

Os testes $t$ e Wilcoxon pareados compararam os resultados clínicos de casos e controles de acordo com a premissa de normalidade. As variáveis categóricas foram comparadas com o teste de McNemar. A normalidade dos dados foi avaliada pelo teste de Shapiro-Wilk. Um valor de $p<0,05$ foi considerado estatisticamente significativo. Todas as análises foram realizadas usando o programa Stata13 (StataCorp, College Station, TX, Estados Unidos).

\section{Resultados}

No total, 68 pacientes foram analisados. Trinta e quatro pacientes foram incluídos em cada grupo ( $\leq 40$ e $>60$ anos); a idade média foi de 30,6 $\pm 6,9$ anos e $65,6 \pm 4,6$ anos nos grupos controle e de casos, respectivamente. A maioria dos pacientes apresentou um defeito do tipo misto com manifestação semelhante de seus sintomas antes da cirurgia. Não houve diferenças no tempo de tração entre os grupos. A - Tabela 1 apresenta uma descrição das características clínicas e demográficas dos dois grupos.

Antes da cirurgia, os pacientes com mais de 60 anos apresentavam diminuição da rotação interna $(p<0,05)$. Não havia diferenças na rotação externa e flexão. Apesar da ausência de mudança do ângulo $\alpha$ entre os grupos, diferenças significativas foram observadas no ângulo LCE (- Tabela 2).

Os resultados funcionais, definidos pela pontuação WOMAC, são resumidos na - Tabela 3. No primeiro ano pósoperatório, houve diferenças nas pontuações de dor, rigidez e funcionalidade entre os dois grupos. 0 grupo com mais de 60 anos de idade apresentou pontuações mais altas em comparação ao grupo controle ( $\leq 40$ anos). À comparação da mudança $(\Delta)$ na pontuação WOMAC entre o período préoperatório e o primeiro ano de acompanhamento nos dois 
Tabela 1 Características demográficas e clínicas dos grupos de casos e controles

\begin{tabular}{|c|c|c|c|}
\hline Variáveis & $\begin{array}{l}\leq 40 \text { Anos } \\
\mathrm{n}=34\end{array}$ & $\begin{array}{l}>60 \text { Anos } \\
\mathrm{n}=34\end{array}$ & Valor de $P$ \\
\hline \multicolumn{4}{|l|}{ Sexo, n (\%) } \\
\hline Feminino & $23(67,6 \%)$ & $23(67,6 \%)$ & \multirow[t]{2}{*}{-} \\
\hline Masculino & $11(32,4 \%)$ & $11(32,4 \%)$ & \\
\hline \multicolumn{4}{|l|}{ Idade (anos) } \\
\hline Média \pm DP & $30,6 \pm 6,9$ & $65,6 \pm 4,6$ & \multirow[t]{2}{*}{-} \\
\hline Variação & $18-40$ & $61-76$ & \\
\hline \multicolumn{4}{|c|}{ Tempo de tração (min) } \\
\hline Média \pm DP & $59,1 \pm 41,1$ & $57,9 \pm 29,6$ & \multirow[t]{2}{*}{0,55} \\
\hline Variação & $16-182$ & $6-131$ & \\
\hline \multicolumn{4}{|l|}{ Lateralidade, n (\%) } \\
\hline Direito & $19(55,9 \%)$ & $18(52,9 \%)$ & \multirow[t]{2}{*}{-} \\
\hline Esquerdo & $15(44,1 \%)$ & $16(47,1 \%)$ & \\
\hline \multicolumn{4}{|l|}{ IFA, n (\%) } \\
\hline Came & $3(8,8 \%)$ & $3(8,8 \%)$ & \multirow[t]{3}{*}{-} \\
\hline Pincer & $4(11,8 \%)$ & $4(11,8 \%)$ & \\
\hline Misto & $27(79,4 \%)$ & $27(79,4 \%)$ & \\
\hline Dor Inguinal, n (\%) & $27(79,4 \%)$ & $30(88,2 \%)$ & 0,54 \\
\hline $\begin{array}{l}\text { Dor ao se sentar, } \\
\mathrm{n}(\%)\end{array}$ & $27(79,4 \%)$ & $31(91,2 \%)$ & 0,28 \\
\hline $\begin{array}{l}\text { Dor ao entrar/ } \\
\text { sair do carro, } \mathrm{n}(\%)\end{array}$ & $22(64,7 \%)$ & $27(79,4 \%)$ & 0,17 \\
\hline
\end{tabular}

Abreviaturas: DP, desvio-padrão; IFA, impacto femoroacetabular; Min, minutos.

grupos, o único quesito com diferenças estatisticamente significativas foi a rigidez. 0 grupo controle ( $\leq 40$ anos) apresentou uma maior alteração na pontuação WOMAC de dor e funcionalidade, sem diferença estatisticamente significativa.

A pontuação WOMAC média de dor, funcionalidade e total sofreu uma diminuição de $79,1 \%, 81,7 \%$ e $81,1 \%$, respectivamente, no grupo controle entre $01^{\circ}$ ano de acompanhamento e o período pré-operatório, em comparação a uma redução de $52,3 \%, 53,7 \%$ e $52,5 \%$, respectivamente, nos pacientes com mais de 60 anos (-Fig. 2A-D).

\section{Complicações}

No grupo com mais de 60 anos, apenas um paciente precisou de revisão artroscópica devido a uma aposição óssea na borda acetabular 1 ano após a cirurgia, correspondendo a $2,9 \%$. Nenhum caso exigiu a realização de artroplastia total do quadril durante esse período de acompanhamento.

\section{Discussão}

O IFA corresponde à principal indicação para a artroscopia do quadril, uma técnica amplamente utilizada na última década. Embora a artroscopia do quadril seja relatada principalmente em populações adultas jovens com resultados satisfatórios, ${ }^{8,10}$
Tabela 2 Resultados pré-operatórios da avaliação física e achados em técnicas de diagnóstico por imagem dos grupos de casos e controles

\begin{tabular}{|c|c|c|c|}
\hline Variáveis & $\begin{array}{l}\leq 40 \text { Anos } \\
n=34\end{array}$ & $\begin{array}{l}>60 \text { Anos } \\
n=34\end{array}$ & $\begin{array}{l}\text { Valor } \\
\text { de } P\end{array}$ \\
\hline \multicolumn{4}{|l|}{ Flexão } \\
\hline Média \pm DP & $121,1^{\circ} \pm 9,1^{\circ}$ & $119,7^{\circ} \pm 10,9^{\circ}$ & \multirow[t]{2}{*}{0,58} \\
\hline Variação & $90^{\circ}-140^{\circ}$ & $90-135$ & \\
\hline \multicolumn{4}{|c|}{ Rotação externa } \\
\hline Média \pm DP & $49,7^{\circ} \pm 18,9^{\circ}$ & $47,1^{\circ} \pm 17,3^{\circ}$ & \multirow[t]{2}{*}{0,45} \\
\hline Variação & $10^{\circ}-80^{\circ}$ & $20^{\circ}-80^{\circ}$ & \\
\hline \multicolumn{4}{|c|}{ Rotação interna } \\
\hline Média \pm DP & $29,5^{\circ} \pm 20,6^{\circ}$ & $22,4^{\circ} \pm 14,7^{\circ}$ & \multirow[t]{2}{*}{$0,04^{*}$} \\
\hline Variação & $-20^{\circ}-70^{\circ}$ & $-10^{\circ}-45^{\circ}$ & \\
\hline \multicolumn{4}{|l|}{ Ângulo alfa } \\
\hline Média \pm DP & $64,4^{\circ} \pm 12,3^{\circ}$ & $64,8^{\circ} \pm 9,4^{\circ}$ & \multirow[t]{2}{*}{0,84} \\
\hline Variação & $42^{\circ}-86^{\circ}$ & $50^{\circ}-86^{\circ}$ & \\
\hline \multicolumn{4}{|l|}{ Ângulo LCE } \\
\hline Média \pm DP & $38,8^{\circ} \pm 6,8^{\circ}$ & $42,1^{\circ} \pm 7,3^{\circ}$ & \multirow[t]{2}{*}{$0,04^{*}$} \\
\hline Variação & $28^{\circ}-53^{\circ}$ & $30^{\circ}-60^{\circ}$ & \\
\hline
\end{tabular}

Abreviaturas: DP, desvio-padrão; IFA, impacto femoroacetabular; LCE, ângulo centro-borda lateral; Min, minutos.

*Valor de $P<0,05$.

estudos recentes sugerem que é uma opção terapêutica viável em populações adultas maduras. ${ }^{6,11}$ Neste estudo, avaliamos os resultados do $1^{\circ}$ ano pós-operatório em uma população acima de 60 anos em comparação a pacientes mais jovens, com até 40 anos de idade. Os resultados mostraram uma melhora na sintomatologia e funcionalidade nos dois grupos. ${ }^{4}$ Isso apoia a tendência atual de oferecimento de opções terapêuticas menos invasivas a pacientes com mais de 60 anos que são funcionalmente ativos e não apresentam alterações degenerativas.

Em nossa coorte de pacientes com mais de 60 anos (idade média: $65,5 \pm 4,6$ anos), observamos uma melhora média de $50 \%$ na escala WOMAC ao longo de 1 ano de acompanhamento. Esses resultados são semelhantes aos de outros autores que avaliaram a funcionalidade com o Harris Hip Score modificado (mHHS, na sigla em inglês). Redmond et al. relataram uma mudança na média do mHHS durante 2 anos de acompanhamento de 63,0 para 80,1 em comparação ao período préoperatório, equivalente a uma melhora de $27 \%{ }^{12}$ Enquanto isso, Mardones et al. ${ }^{13}$ incluíram 28 pacientes com idade média de $64,3 \pm 5,1$ anos e relataram uma melhora de $47 \%$ a partir do valor mediano pré-operatório de 53 pontos no mHHS. Outros estudos, como os de Philippon et al. ${ }^{6}$ e Ben Tov et al., ${ }^{5}$ também relataram resultados satisfatórios em uma população adulta com 50 anos ou mais.

Domb et al. ${ }^{7}$ avaliaram e compararam os resultados funcionais de pacientes com mais de 50 anos pareados com adultos de menos de 30 anos de idade por um período de acompanhamento de 2 anos; esses autores determinaram que não há diferenças no mHHS entre os 2 grupos de 


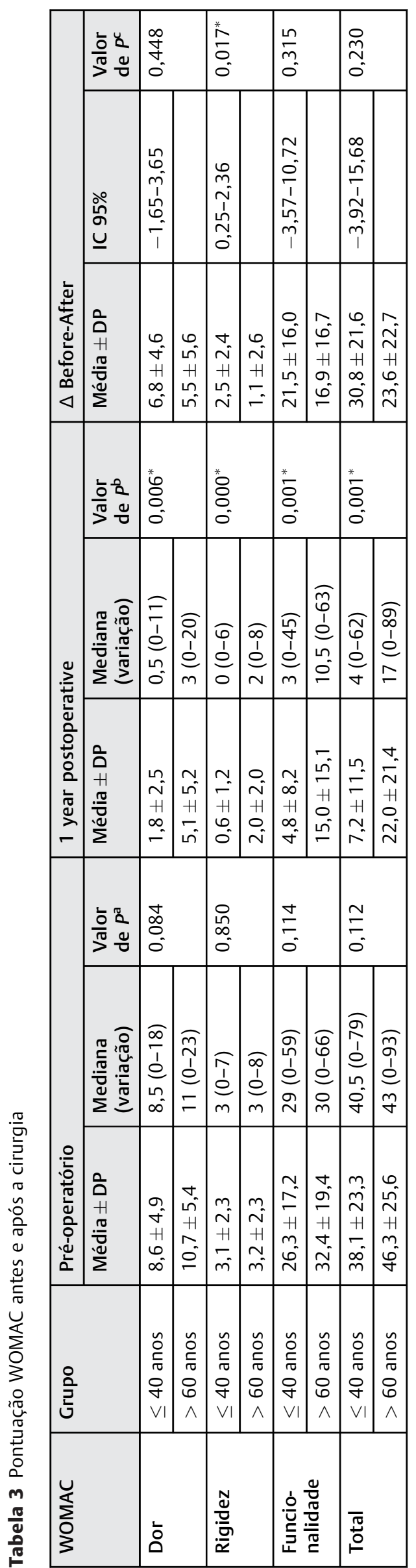

pacientes ( $>50$ anos: $82,2 \pm 16,2 ;<30$ anos: $84,2 \pm 19,1$, valor de $p>0,05$ ). Isso sugere que o tratamento artroscópico pode ser benéfico para adultos com mais de 50 anos. Em nosso estudo, não observamos diferença estatisticamente significativa entre os grupos no acompanhamento por um ano. No entanto, a relevância clínica, com melhora média de $81,1 \%$ na pontuação WOMAC em pacientes com até 40 anos em comparação aos pacientes mais velhos, foi observada. Essas diferenças entre os dois grupos podem estar associadas ao envelhecimento e seu impacto na capacidade funcional e na reabilitação. McCormick et al. ${ }^{14}$ demonstraram um efeito semelhante em adultos com menos de 40 anos com resultados funcionais melhores.

Na população adulta, especificamente com mais de 50 anos, os resultados pós-operatórios dependem principalmente da seleção adequada dos pacientes. As lesões condrais e a OA avançada são consideradas fatores prognósticos negativos para os resultados clínicos. ${ }^{4,15}$ Um estudo prospectivo de Byrd et al., ${ }^{16}$ com 10 anos de acompanhamento de uma coorte de 50 pacientes submetidos à artroscopia do quadril, revelou resultados insatisfatórios nos casos com evidência de OA no momento da intervenção (14 quadris). Como a artroplastia total do quadril (ATQ) foi necessária para a melhora dos sintomas em 78,6\% desses quadris, a presença de OA foi considerada um fator de mau prognóstico. Menge et al., ${ }^{17}$ em um acompanhamento de longo prazo de 145 pacientes, observou uma taxa de conversão em ATQ de $34 \%$, com idade média de 53 anos no momento da cirurgia; no entanto, nenhum dos pacientes com menos de 35 anos precisou de ATQ. Os autores relataram que a taxa de conversão era maior em pacientes com idade avançada, espaço articular $<2 \mathrm{~mm}$ e microfratura acetabular. Resultados anteriores motivaram a comunidade médica a examinar a seleção de pacientes para artroscopia do quadril em populações maduras. Em nossa experiência, não operávamos pacientes com mais de 60 anos e alterações degenerativas avançadas, traumas anteriores ou comorbidades, como distúrbios peritrocantéricos ou neurológicos.

Em nossa coorte, a taxa de revisão foi de 2,9\% (um caso) no grupo mais velho pelo período de acompanhamento de 12 meses. Degen et al., ${ }^{18}$ em um acompanhamento de 2 anos, descreveram que apenas 3,8\% dos 8.267 procedimentos foram submetidos à revisão artroscópica; além disso, identificaram a idade superior a 50 anos como fator de risco (razão de risco: 2,09 [intervalo de confiança de 95\%: 1,82-2,39; valor de $p<0,01]$ ).

Entre os pontos fortes deste estudo, em $1^{\circ}$ lugar, está o processo de pareamento da população acima de 60 anos e mais jovens ( $\leq 40$ anos), realizada de acordo com o tipo de IFA, os procedimentos, e o tempo de evolução clínica desde a cirurgia. Esse processo permitiu o controle de fatores que poderiam influenciar os resultados funcionais do paciente, como a técnica cirúrgica e a curva de aprendizado do cirurgião ortopédico. Todos os casos foram realizados pela mesma equipe cirúrgica (cirurgião e anestesiologista) em um centro de referência em preservação do quadril, o que não permite a generalização direta dos resultados de outras instituições. Portanto, os leitores devem interpretar nossas 

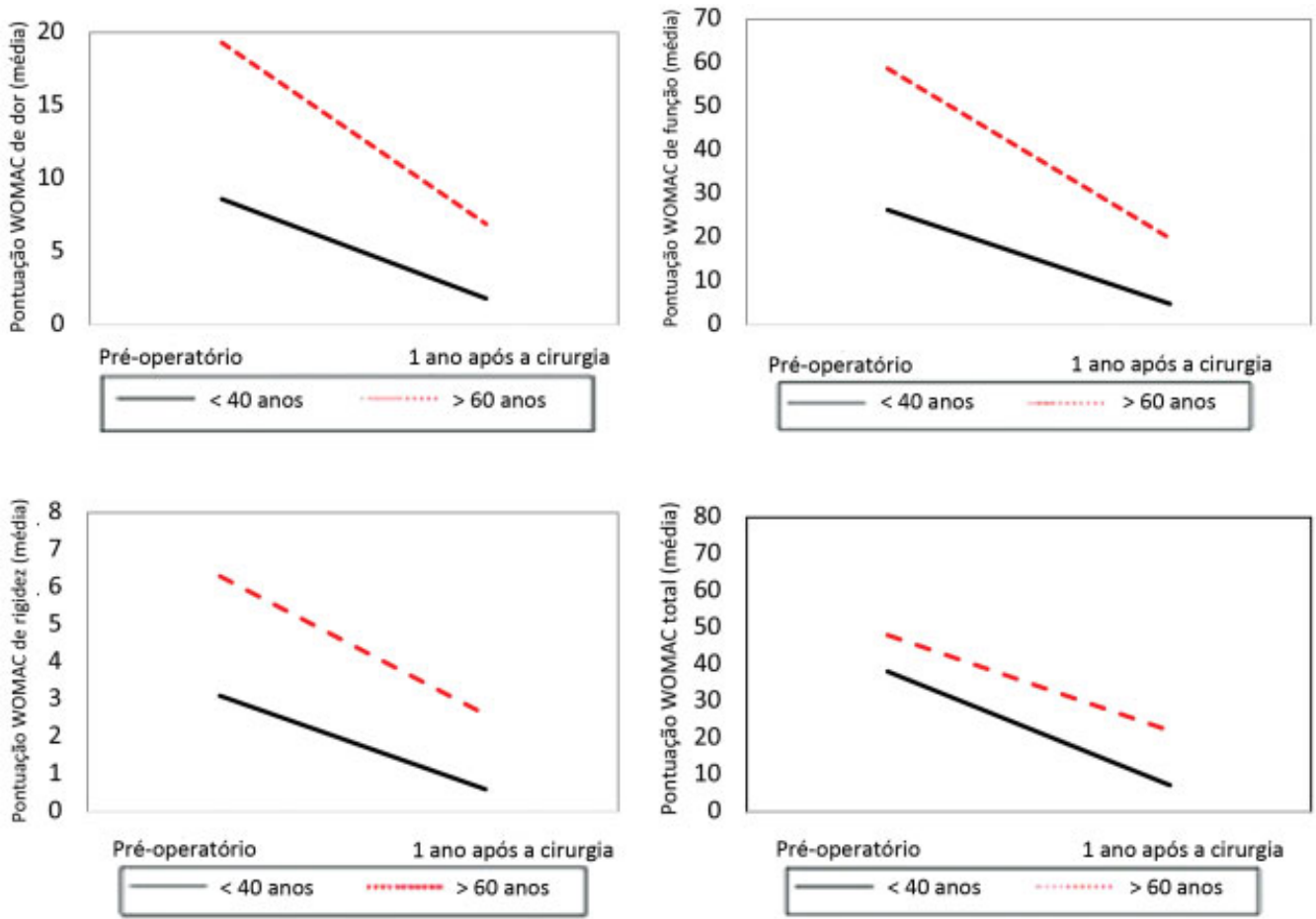

Fig. 2 Alteração na pontuação WOMAC entre o período pré-operatório e 1 ano após a cirurgia.

descobertas com cautela, de acordo com as características dos pacientes e os critérios médicos.

Em segundo lugar, a exclusão de pacientes com um certo grau de $\mathrm{OA}$ avançada ou outras patologias do quadril permitiu a descrição objetiva dos resultados clínicos da artroscopia do quadril na população madura. Embora nosso tempo de acompanhamento tenha sido curto, ele permitiu a avaliação simultânea dos resultados clínicos em pacientes mais de 60 anos e menos de 40 anos de idade. A pontuação WOMAC foi a única utilizada na avaliação do grau de dor e funcionalidade, mas não permitiu a determinação precisa do grau de atividade física após o procedimento e o nível de satisfação. Além disso, como essa pontuação não foi utilizada na maioria dos estudos, a comparação direta com outras coortes talvez não seja possível. Embora WOMAC não seja a melhor escala para avaliação dos resultados funcionais no campo da cirurgia de preservação do quadril, acreditamos que permita a determinação de resultados funcionais da perspectiva do paciente.

Mais estudos em longo prazo para quantificação das taxas de revisão cirúrgica e conversão em ATQ na população madura com critérios de seleção adequados para artroscopia do quadril são essenciais.

\section{Conclusão}

O grupo de pacientes com 40 anos ou menos apresentou uma mudança considerável na pontuação WOMAC, mas sem significado estatístico em comparação ao grupo acima de 60 anos. Isso sugere que a artroscopia do quadril é benéfica em caso de uma seleção apropriada de pacientes com IFA, independentemente da idade dos indivíduos.
Conflito de Interesses

Os autores declaram não haver conflito de interesses.

\section{Agradecimento}

Agradecemos ao Instituto de Pesquisa do Centro Médico Imbanaco por seu apoio durante o desenvolvimento deste projeto. Os autores também são gratos a Evelyn Bedoya por seu auxílio com a análise estatística.

\section{Referências}

1 Ganz R, Leunig M, Leunig-Ganz K, Harris WH. The etiology of osteoarthritis of the hip: an integrated mechanical concept. Clin Orthop Relat Res 2008;466(02):264-272

2 Leunig M, Ganz R. [Femoroacetabular impingement. A common cause of hip complaints leading to arthrosis]. Unfallchirurg 2005; 108(01):9-10, 12-17

3 Murphy NJ, Eyles JP, Hunter DJ. Hip Osteoarthritis: Etiopathogenesis and Implications for Management. Adv Ther 2016;33(11): 1921-1946

4 Griffin DW, Kinnard MJ, Formby PM, McCabe MP, Anderson TD. Outcomes of Hip Arthroscopy in the Older Adult: A Systematic Review of the Literature. Am J Sports Med 2017;45(08):1928-1936

5 Ben Tov T, Amar E, Shapira A, Steinberg E, Atoun E, Rath E. Clinical and functional outcome after acetabular labral repair in patients aged older than 50 years. Arthroscopy 2014;30(03):305-310

6 Philippon MJ, Schroder E Souza BG, Briggs KK, Briggs KK. Hip arthroscopy for femoroacetabular impingement in patients aged 50 years or older. Arthroscopy 2012;28(01):59-65

7 Domb BG, Linder D, Finley Z, et al. Outcomes of hip arthroscopy in patients aged 50 years or older compared with a matched-pair control of patients aged 30 years or younger. Arthroscopy 2015; 31(02):231-238

8 Bryan AJ, Krych AJ, Pareek A, Reardon PJ, Berardelli R, Levy BA. Are Short-term Outcomes of Hip Arthroscopy in Patients 55 Years and 
Older Inferior to Those in Younger Patients? Am J Sports Med 2016;44(10):2526-2530

9 Aguilera-Bohorquez B, Gil E, Fonseca J, Fernandez M, Sánchez M. Tenosuspension of the Reflected Head of the Rectus Femoris in Hip Arthroscopy: Description of a Portal and a Surgical Maneuver. Arthrosc Tech 2017;6(04):e1015-e1019

10 Ganz R, Parvizi J, Beck M, Leunig M, Nötzli H, Siebenrock KA. Femoroacetabular impingement: a cause for osteoarthritis of the hip. Clin Orthop Relat Res 2003;(417):112-120

11 Javed A, O’Donnell JM. Arthroscopic femoral osteochondroplasty for cam femoroacetabular impingement in patients over 60 years of age. J Bone Joint Surg Br 2011;93(03):326-331

12 Redmond JM, Gupta A, Cregar WM, Hammarstedt JE, Gui C, Domb BG. Arthroscopic Treatment of Labral Tears in Patients Aged 60 Years or Older. Arthroscopy 2015;31(10):1921-1927

13 Mardones R, Via AG, Rivera A, et al. Arthroscopic treatment of femoroacetabular impingement in patients older than 60 years. Muscles Ligaments Tendons J 2016;6(03):397-401
14 McCormick F, Nwachukwu BU, Alpaugh K, Martin SD. Predictors of hip arthroscopy outcomes for labral tears at minimum 2-year follow-up: the influence of age and arthritis. Arthroscopy 2012;28 (10):1359-1364

15 Horner NS, Ekhtiari S, Simunovic N, Safran MR, Philippon MJ, Ayeni OR. Hip Arthroscopy in Patients Age 40 or Older: A Systematic Review. Arthroscopy 2017;33(02):464-475.e3

16 Byrd JWT, Jones KS. Prospective analysis of hip arthroscopy with 10-year followup. Clin Orthop Relat Res 2010;468(03): 741-746

17 Menge TJ, Briggs KK, Dornan GJ, McNamara SC, Philippon MJ. Survivorship and Outcomes 10 Years Following Hip Arthroscopy for Femoroacetabular Impingement: Labral Debridement Compared with Labral Repair. J Bone Joint Surg Am 2017;99(12): 997-1004

18 Degen RM, Pan TJ, Chang B, et al. Risk of failure of primary hip arthroscopy-a population-based study. J Hip Preserv Surg 2017;4 (03):214-223 